\title{
Effects of acute kidney dysfunction on hypothalamic arginine vasopressin synthesis in transgenic rats
}

\author{
Hiromichi Ueno ${ }^{1,2} \cdot$ Ryota Serino $^{3} \cdot$ Kenya Sanada $^{1,2} \cdot$ Yasuki Akiyama $^{1} \cdot$ Kentaro Tanaka $^{1} \cdot$ Haruki Nishimura $^{1}$. \\ Kazuaki Nishimura ${ }^{1} \cdot$ Satomi Sonoda ${ }^{1}$. Yasuhito Motojima ${ }^{1} \cdot$ Reiko Saito $^{1} \cdot$ Mitsuhiro Yoshimura $^{1}$. \\ Takashi Maruyama $^{1} \cdot$ Tetsu Miyamoto $^{2} \cdot$ Masahito Tamura $^{2} \cdot$ Yutaka Otsuji $^{2} \cdot$ Yoichi Ueta $^{1}$ (])
}

Received: 8 November 2018 / Accepted: 25 March 2019 / Published online: 1 April 2019

(c) The Physiological Society of Japan and Springer Japan KK, part of Springer Nature 2019

\begin{abstract}
Acute loss of kidney function is a critical internal stressor. Arginine vasopressin (AVP) present in the parvocellular division of the paraventricular nucleus (PVN) plays a key role in the regulation of stress responses. However, hypothalamic AVP dynamics during acute kidney dysfunction remain unclear. In this study, we investigated the effects of bilateral nephrectomy on AVP, using a transgenic rat line that expressed the AVP-enhanced green fluorescent protein (eGFP). The eGFP fluorescent intensities in the PVN were dramatically increased after bilateral nephrectomy. The mRNA levels of $e G F P, A V P$, and corticotrophin-releasing hormone in the PVN were dramatically increased after bilateral nephrectomy. Bilateral nephrectomy also increased the levels of Fos-like immunoreactive cells in brainstem neurons. These results indicate that bilateral nephrectomy upregulates the AVP-eGFP synthesis. Further studies are needed to identify the neural and/or humoral factors that activate AVP synthesis and regulate neuronal circuits during acute kidney dysfunction.
\end{abstract}

Keywords Vasopressin $\cdot$ Transgenic rat $\cdot$ In situ hybridization $\cdot$ Hypothalamus $\cdot$ Bilateral nephrectomy

\section{Introduction}

Stress is a state of real or perceived threat to homeostasis. It is important to adapt internal and external stressors for the maintenance of homeostasis to ensure the survival and longevity of an organism. Exposure to various stressors activates the stress response, which is mediated by a complex interaction between the central nervous and peripheral systems including the autonomic nervous system, endocrine

Electronic supplementary material The online version of this article (https://doi.org/10.1007/s12576-019-00675-8) contains supplementary material, which is available to authorized users.

Yoichi Ueta

yoichi@med.uoeh-u.ac.jp

1 Department of Physiology, School of Medicine, University of Occupational and Environmental Health, 1-1 Iseigaoka, Yahatanishi-ku, Kitakyushu 807-8555, Japan

2 The Second Department of Internal Medicine, School of Medicine, University of Occupational and Environmental Health, Kitakyushu 807-8555, Japan

3 Department of Nephrology, Yoshino Hospital, Kitakyushu 808-0034, Japan system, and immune system. The paraventricular nucleus (PVN) of the hypothalamus is one of the integrative sites of the autonomic nervous system and neuroendocrine system that deals with various aversive stressors. Arginine vasopressin (AVP) and corticotropin-releasing hormone (CRH), which are both synthesized in the parvocellular neurons of the PVN (pPVN), play important roles in the regulation of stress responses by activating the hypothalamic-pituitary-adrenal (HPA) axis. The functions of AVP, which maintain water homeostasis by regulating the water reabsorption in the renal collecting duct, is well known [1]. In addition, central AVP has many physiological functions as a neurotransmitter, such as stress response, social behavior, and circadian rhythm [2-5]. In particular, stressinduced activation of HPA axis is thought to be important in maintaining homeostasis in the whole body for survival. Acute immobilization stress leads to the upregulation of $A V P$ and $C R H$ mRNA expression in the pPVN [6]. Most AVP is synthesized in the PVN magnocellular division (mPVN) and the supraoptic nucleus (SON) of the hypothalamus [4, 7]. In addition, AVP neurons also exist in the suprachiasmatic nucleus ( $\mathrm{SCN}$ ), which is responsible for controlling circadian rhythms [4, 5]. Hypothalamic AVP synthesis and 
release are mainly regulated by several neurohumoral factors and the autonomic nervous system.

The kidney has important functions that maintain homeostasis in the body by regulating the fluid and electrolyte balance. Therefore, loss of kidney function is a critical stressor for the entire body. Our team has investigated the central AVP changes under various stress conditions [2, 3, 8, 9]. However, the central AVP dynamics under stress conditions induced by bilateral nephrectomy remain unclear. In this study, we first examined bilateral nephrectomy-induced changes of AVP in the PVN, SON, and SCN, using a transgenic rat line that expressed the AVP-enhanced green fluorescent protein (eGFP), which is used as a quantitative reporter of AVP expression [2, 3, 8, 10]. Second, the gene expression of $e G F P, A V P$, and $C R H$ in the hypothalamus after bilateral nephrectomy was evaluated. Finally, we counted the number of Fos-like immunoreactive (LI) cells in the locus coeruleus (LC), area postrema (AP), nucleus tractus solitarius (NTS), and rostral ventrolateral medulla (RVLM), which are involved in the regulation of biological responses to severe stressors by controlling AVP synthesis and the modulation of sympathetic nerve activity [7, 11-13].

\section{Materials and methods}

\section{Animals}

Adult male AVP-eGFP transgenic rats weighing 200-300 g were used. All rats were housed in conditions of three animals per cage and maintained in an air-conditioned room $\left(23-25^{\circ} \mathrm{C}, 12: 12\right.$ light/dark cycle, lights on at 7:00 am) with food and water available ad libitum. All rats were screened by polymerase chain reaction analysis of genomic DNA extracted from rat ear biopsies, as previously described. All experiments were performed in accordance with the guidelines on the use and care of the laboratory animals as set out care by the Physiological Society of Japan and under control of the Ethics Committee of Animal Care and Experimentation, University of Occupational and Environmental Health (Fukuoka, Japan).

\section{Bilateral nephrectomy}

Before all surgeries, the transgenic rats were anesthetized by intraperitoneal (i.p.) administration of the mixture of three anesthetic agents, namely butorphanol $(5.0 \mathrm{mg} / \mathrm{kg})$, midazolam $(4.0 \mathrm{mg} / \mathrm{kg})$, and medetomidine $(0.3 \mathrm{mg} / \mathrm{kg})$ [14]. The transgenic rats were either bilaterally nephrectomized or had sham surgery; the bilateral kidney was identified and left intact.

\section{Measurement of eGFP fluorescence in the hypothalamus}

To analyze the changes in AVP-eGFP and Fos expression over time, transgenic rats were transcardially perfused at 3 , 12 , and $20 \mathrm{~h}$ after operation at 10:00-11:00 $(n=6$ in each group at each time point). The rats were deeply anesthetized by i.p. injection of the mixture of three anesthetic agents. Rats were transcardially perfused with $0.1 \mathrm{M}$ phosphate buffer (PB, pH 7.4) containing heparin (1000 U/l) and 4\% paraformaldehyde in $0.1 \mathrm{M}$ PB $[8,15]$. The brain tissues were post-fixed in $4 \%$ paraformaldehyde in $0.1 \mathrm{M} \mathrm{PB}$ for 2 days at $4{ }^{\circ} \mathrm{C}$ and immersed in $20 \%$ sucrose in $0.1 \mathrm{M}$ PB for 2 days. Then the tissues were cut at a thickness of $40 \mu \mathrm{m}$ with a microtome (REM-700; Yamato Kohki Industrial Co., Ltd., Saitama, Japan). We observed and analyzed eGFP fluorescence in the PVN, SON, and SCN by fluorescence microscopy (ECLIPSE Ti-E; Nikon, Tokyo, Japan) with a GFP filter (Nikon) and imaging analysis system (NIS-Elements; Nikon), as previously described [3, 8]. The observation regions were selected by the rat brain atlas [16].

\section{Immunochemistry of c-Fos in the brainstem regions}

As previously described, we performed immunochemistry for c-Fos [1, 17-19]. The free-floating sections were incubated for 3 days with a 1:500 dilution [in phosphate-buffered saline (PBS)] of primary rabbit polyclonal anti-Fos antibody (\#sc-52; Santa Cruz Biotechnology, Dallas, TX, USA). After washing, sections were incubated for $2 \mathrm{~h}$ with a 1:1000 dilution of Alexa Flour 546 goat anti-rabbit IgG secondary antibody (\#A11035; Molecular Probes, Eugene, OR, USA). We observed the sections by fluorescence microscopy with a red fluorescence protein filter (Nikon) to observe the Fos-LI cells. The observation areas were determined by the rat brain atlas [16], as described above. Then, we manually counted the number of Fos-LI-positive nuclei in the LC, AP, NTS, and RVLM.

\section{In situ hybridization for eGFP, AVP, and CRH mRNA}

To analyze the changes in $e G F P, A V P$, and $C R H$ levels over time in AVP-eGFP, transgenic rats were decapitated at 3,12, and $20 \mathrm{~h}$ after surgery at 10:00-11:00 $(n=6$ in each group at each time point) as described above, followed by immunochemistry $[1,8,9]$. In brief, the brains were removed, frozen, and stored. Blood samples were collected to measure the plasma concentrations of potassium $\left(\mathrm{K}^{+}\right)$, blood urea nitrogen (BUN), and creatinine (Cre). The frozen brain tissues were cut at a thickness of $12 \mu \mathrm{m}$ with a cryostat at $-20^{\circ} \mathrm{C}$. The observation regions were selected by the rat brain atlas 
[16]. The probe sequence used for in situ hybridization is shown in Table 1. The reliability of the probes has been previously described [1, 20, 21]. Hybridized sections were exposed to autoradiography film (Amersham Hyperfilm, Buckinghamshire, UK) for 5 days for $e G F P ; 8 \mathrm{~h}$ for $A V P$ in the mPVN, SON, and SCN; 2 days for $A V P$ in the pPVN; and 3 days for $C R H$. Analysis of the autoradiography images was conducted using the MCID system (Imaging Research, Inc., St. Catherines, ON, Canada). Kinoshita et al. showed the methods using a threshold tool image analysis program to determine changes in AVP mRNA expression in the pPVN [22].

\section{Statistical analysis}

All data are presented as mean \pm standard error of the mean (SEM). Statistical analysis was performed using one-way analysis followed by the Bonferroni post hoc test for multiple comparisons. $P<0.05$ was considered statistically significant.

\section{Results}

\section{Plasma concentrations of $\mathrm{K}^{+}, \mathrm{BUN}$, and Cre}

The plasma concentrations of $\mathrm{K}^{+}$, BUN, and Cre are shown in Table 2. They markedly increased over after bilateral nephrectomy (Table 2).

\section{eGFP intensity in the hypothalamus}

Representative digital images of eGFP fluorescent intensity in the PVN (Fig. 1A), SON (Fig. 1B), and SCN (Fig. 1C) of untreated (Fig. 1A-C-a), sham-operated (Fig. 1A-C-b-d), and bilaterally nephrectomized (Fig. 1A-C-f-h) rats were observed at 3 (b, f), 12 (c, g), and 20 h (d, h). Strong eGFP fluorescence could be seen in the pPVN at 12 and $20 \mathrm{~h}$ after bilateral nephrectomy (Fig. 1A-g, h). The ratio of eGFP fluorescence significantly increased (Fig. 2A-a) at 12 and $20 \mathrm{~h}$ after bilateral nephrectomy. There were no significant differences in the mPVN (Fig. 2A-b), SON (Fig. 2B), and SCN (Fig. 2C) after bilateral nephrectomy.
Table 2 Effects of i.p. bilateral nephrectomy $(\mathrm{Nx})$ or sham operation on plasma concentrations of potassium $\left(\mathrm{K}^{+}\right)$, blood urea nitrogen (BUN), and creatinine (Cre) at 3,12 , and $20 \mathrm{~h}$ after treatment

\begin{tabular}{|c|c|c|c|}
\hline $\begin{array}{l}\text { Time after treatments } \\
\text { (h) }\end{array}$ & 3 & 12 & 20 \\
\hline \multicolumn{4}{|c|}{ Plasma concentration of $\mathrm{K}^{+}(\mathrm{mEq} / \mathrm{l})$} \\
\hline Sham operation & $8.1 \pm 0.4$ & $6.9 \pm 0.3$ & $7.0 \pm 0.3$ \\
\hline Bilateral nephrectomy & $8.6 \pm 0.3$ & $8.5 \pm 0.4^{* *}$ & $10.2 \pm 0.6^{* *}$ \\
\hline \multicolumn{4}{|c|}{ Plasma concentration of BUN (mg/dl) } \\
\hline Sham operation & $34.6 \pm 5.8$ & $20.3 \pm 1.5$ & $17.7 \pm 0.7$ \\
\hline Bilateral nephrectomy & $48.8 \pm 2.0$ & $94.9 \pm 2.4 * *$ & $123.8 \pm 2.9 * *$ \\
\hline \multicolumn{4}{|c|}{ Plasma concentration of Cre (mg/dl) } \\
\hline Sham operation & $0.23 \pm 0.02$ & $0.22 \pm 0.01$ & $0.23 \pm 0.02$ \\
\hline Bilateral nephrectomy & $0.66 \pm 0.03 * *$ & $1.58 \pm 0.16^{* *}$ & $2.7 \pm 0.02 * *$ \\
\hline
\end{tabular}

Data are presented as the mean \pm SEM (each group at each time point, $n=6)$

$* P<0.05, * * P<0.01$ compared with the saline-treated group. Plasma $\mathrm{K}^{+}, \mathrm{BUN}$, and Cre in untreated rats $(n=6)$ were $8.6 \pm 0.1 \mathrm{mEq} / \mathrm{L}$, $17.4 \pm 0.5 \mathrm{mg} / \mathrm{dl}$, and $0.25 \pm 0.01 \mathrm{mg} / \mathrm{dl}$ respectively

\section{Gene expression of GFP gene in the hypothalamus}

Representative images of $G F P$ gene expression were taken in the PVN (Fig. 3A), SON (Fig. 3B), and SCN (Fig. 3C) from untreated (Fig. 3A-C-a), sham-operated (Fig. 3A-C-b, c, d), and bilaterally nephrectomized (Fig. 3A-C-f-h) rats at 3, 12, and $20 \mathrm{~h}$ post-surgery. The GFP levels markedly increased in the pPVN at 12 and $20 \mathrm{~h}$ after bilateral nephrectomy compared with sham-operated rats (Fig. 6A-a). There were no significant differences in GFP levels in the mPVN, $\mathrm{SON}$, and SCN among the three groups at all time points (Fig. 6A-b-d).

\section{Gene expression of AVP in the hypothalamus}

Representative images of $A V P$ gene expression were taken in the PVN (Fig. 4A), SON (Fig. 4B), and SCN (Fig. 4C) from untreated (Fig. 4A-C-a), sham-operated (Fig. 4A-C-b-d), and bilaterally nephrectomized (Fig. 4A-C-f-h) rats at 3, 12, and $20 \mathrm{~h}$ post-surgery. The $A V P$ levels markedly increased in the pPVN at 12 and $20 \mathrm{~h}$ after bilateral nephrectomy compared with sham-operated rats (Fig. 6B-a). There were no significant differences in $A V P$ levels in the mPVN, SON, and $\mathrm{SCN}$ in the three groups at all time points (Fig. 6B-b-d).
Table 1 The probe sequence for in situ hybridization

\begin{tabular}{ll}
\hline Probe & Sequence \\
\hline$e G F P$ & 5'-CGG CCA TGA TAT AGA CGT TGT GGC TGT TGT AGT TGT ACT CC-3' \\
$A V P$ & 5'-CAG CTC CCG GGC TGG CCC GTC CAG CT-3' \\
$C R H$ & 5'-CAG TTT CCT GTT GCT GTG AGC TTG CTG AGC TAA CTG CTC TGC \\
& CCT GGC-3' \\
\hline
\end{tabular}




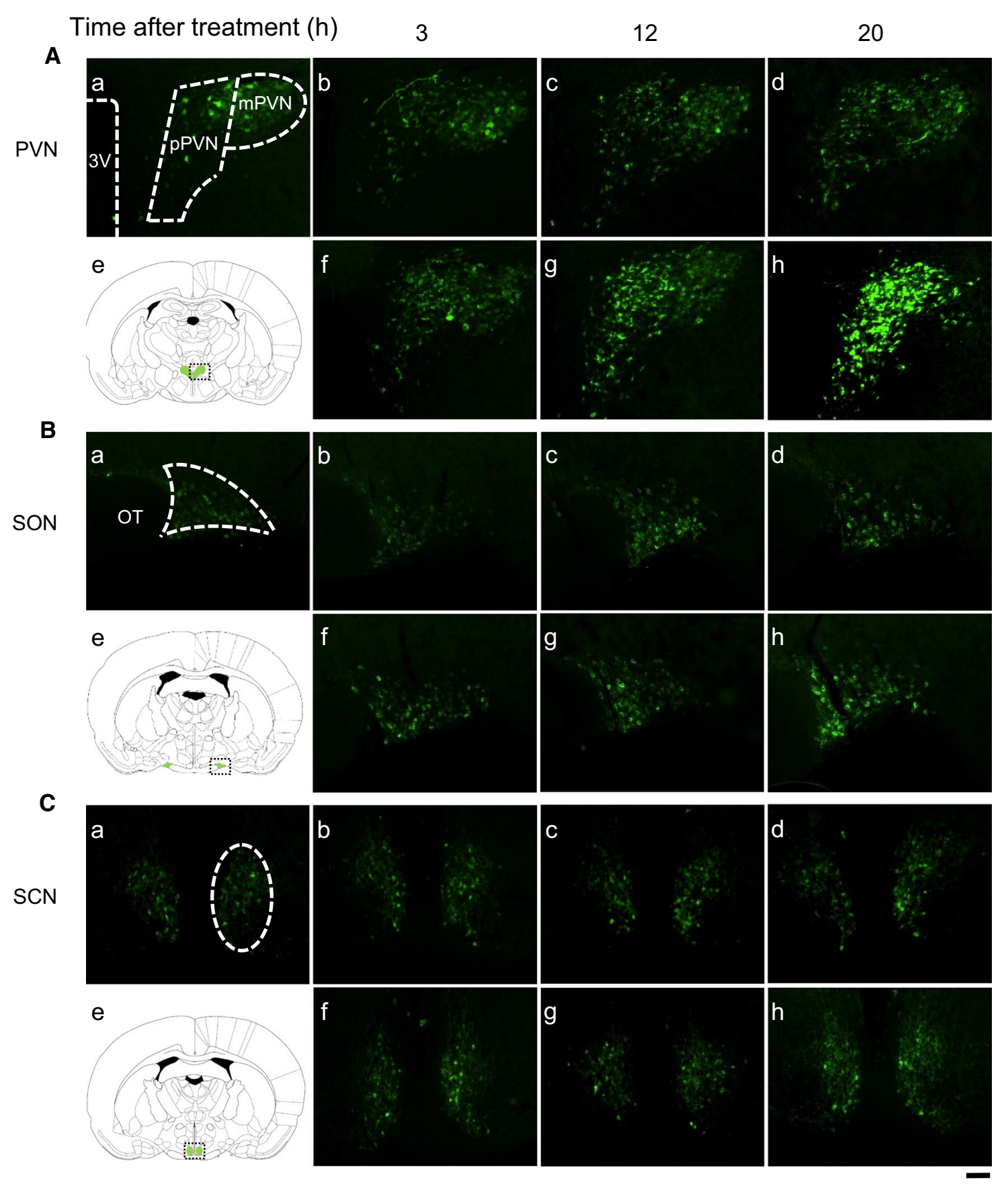

Fig. 1 Effects of bilateral nephrectomy $(\mathrm{Nx})$ or sham operation (Sham) on eGFP in the PVN (A), SON (B), and SCN (C) in AVPeGFP transgenic rats. Sections were obtained from untreated (A-Ca), sham-operated (A-C-b-d), and bilateral-nephrectomized rats (A-C-f-h) at 3, 12, and $20 \mathrm{~h}$ after treatment (each group at each time

\section{Gene expression of $\mathrm{CRH}$ in the PVN}

Representative images of $A V P$ gene expression were taken in the pPVN of untreated (Fig. 5a), sham-operated (Fig. 5b-d), and bilaterally nephrectomized (Fig. 5f, g, h) rats at 3,12 , and $20 \mathrm{~h}$ after operation. The $C R H$ levels point, $n=6$ ). Schematic anatomic representation of the nuclei (green shading) adapted from the rat brain atlas (A-C-e). $O T$ optic tract, $3 \mathrm{~V}$ third ventricle, $p P V N$ parvocellular division of $\mathrm{PVN}, m P V N$ magnocellular division of PVN. Scale bar $100 \mu \mathrm{m}$

markedly increased in the pPVN at 12 and $20 \mathrm{~h}$ after bilateral nephrectomy compared with sham-operated rats (Fig. 6C). 


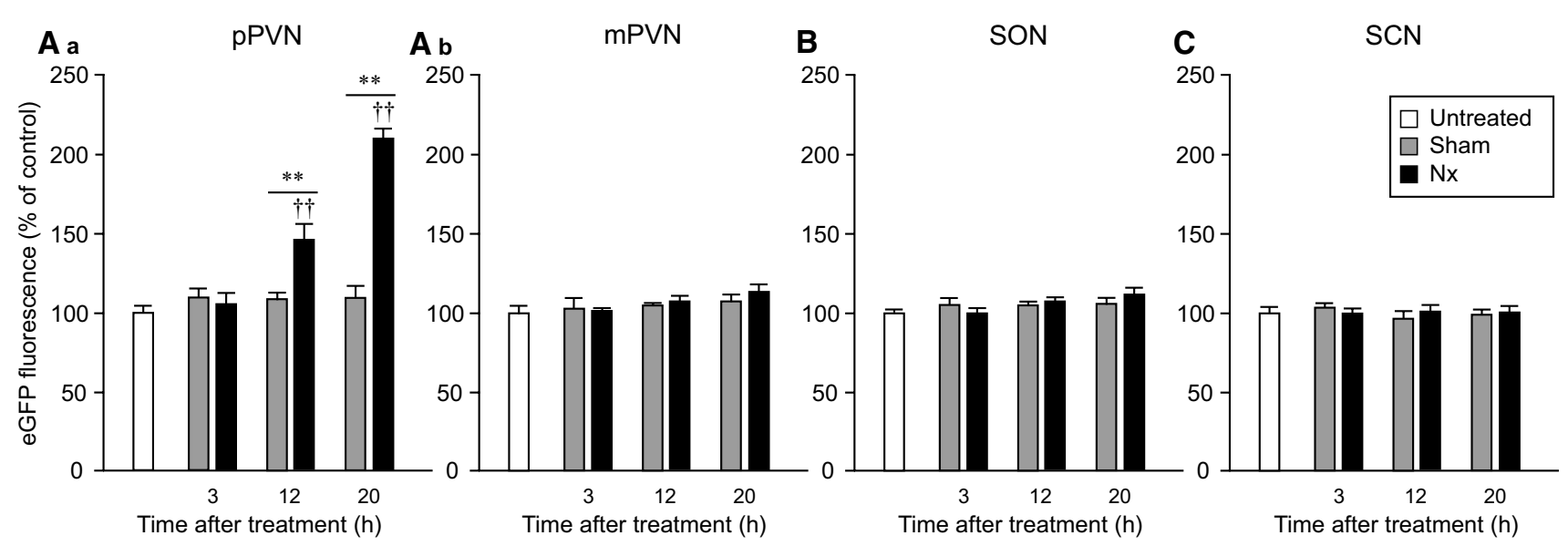

Fig. 2 eGFP fluorescence intensity in the PVN (A-a; pPVN, -b; $\mathrm{mPVN}$ ), and SCN (C) at 3, 12, and $20 \mathrm{~h}$ after bilateral nephrectomy $(\mathrm{Nx})$ or sham operation (Sham). Data are presented as the

\section{Expression patterns of Fos-LI cells in the brain stem neurons are responsible for the control of AVP synthesis}

Representative images of c-Fos-LI cells were taken in the LC (Fig. 7A), AP (Fig. 7B), NTS (Fig. 7C), and RVLM (Fig. 7D) from untreated (Fig. 7A-D-a), sham-operated (Fig. 7A-D-b-d), and bilaterally nephrectomized (Fig. 7A-D-f-h) rats at 3 (b, f), 12 (c, g), and $20 \mathrm{~h}(\mathrm{~d}, \mathrm{~h})$. The number of Fos-LI cells in the LC (Fig. 8A), AP (Fig. 8B), NTS (Fig. 8C), and RVLM (Fig. 8D) after bilateral nephrectomy was significantly increased compared with the shamoperated group.

\section{Discussion}

A transgenic rat line expressing AVP-enhanced green fluorescent protein enabled us to evaluate hypothalamic AVP changes by observing eGFP fluorescence [2, 9, 10, 23]. First, we observed the upregulation of AVP-eGFP after bilateral nephrectomy in the pPVN. Second, our data clearly showed that $e G F P, A V P$, and $C R H$ mRNA levels in the pPVN after bilateral nephrectomy were dramatically increased. Finally, the number of Fos-LI cells in the LC, AP, NTS, and RVLM were significantly increased after bilateral nephrectomy. To the best of our knowledge, this is the first report to reveal an increase in hypothalamic AVP synthesis after bilateral nephrectomy.

In this study, we revealed the upregulation of AVP synthesis in the pPVN after bilateral nephrectomy by analyzing eGFP fluorescence and $e G F P$ gene transcription. In general, the synthesis of AVP in the pPVN does not reflect plasma AVP levels, because AVP synthesized in the pPVN is not mean \pm SEM (each group at each time point, $n=6$ ). ${ }^{\dagger} P<0.05$, ${ }^{\dagger \dagger} P<0.01$ compared with the untreated group. $* P<0.05$, $* * P<0.01$ compared with the sham-operated group

secreted into the systemic circulation [24]. Thus, analyzing fluorescence and gene transcription are important methods for evaluating AVP synthesis of pPVN. Upregulation of $A V P$ and $C R H$ expression in pPVN was expected to activate adrenal function via HPA axis. This adrenal hyperactivity could cause various complications (immunodeficiency, osteoporosis, and hypertension) related to kidney dysfunction. Therefore, it is important to reveal the hypothalamic AVP dynamics after bilateral nephrectomy to understand the mechanisms underlying biological responses to severe stress induced by kidney dysfunction. In the present study, we have focused on hypothalamic AVP change in acute phase as the first step in research on the central effect of stress induced by kidney dysfunction. However, the complications are often a problem mainly in the chronic phase of kidney dysfunction. Further studies are needed in order to clarify whether the upregulation of $A V P$ and $C R H$ keep for a long time under kidney dysfunction or not.

In previous reports, we revealed that dramatic increases in hypothalamic $e G F P$ levels compared with endogenous $A V P$ levels in several stress conditions by using AVP-eGFP rats $[2,3,8]$. Thus, we could detect small changes that were undetectable in wild-type rats by observing the changes in AVP-eGFP. In particular, this property helps to evaluate the change in AVP of pPVN, since the problem that endogenous $A V P$ expression of pPVN is much smaller than that of mPVN makes analysis of AVP synthesis in pPVN difficult. Indeed, eGFP fluorescence and $e G F P$ expression responded robustly to bilateral nephrectomy in this study. In addition, we have previously shown that there were no differences in plasma AVP level and $A V P$-mRNA level between wild-type and transgenic rats under normal conditions and salt-loaded conditions [9]. From this result, the change of endogenous AVP after bilateral nephrectomy in AVP-eGFP transgenic 


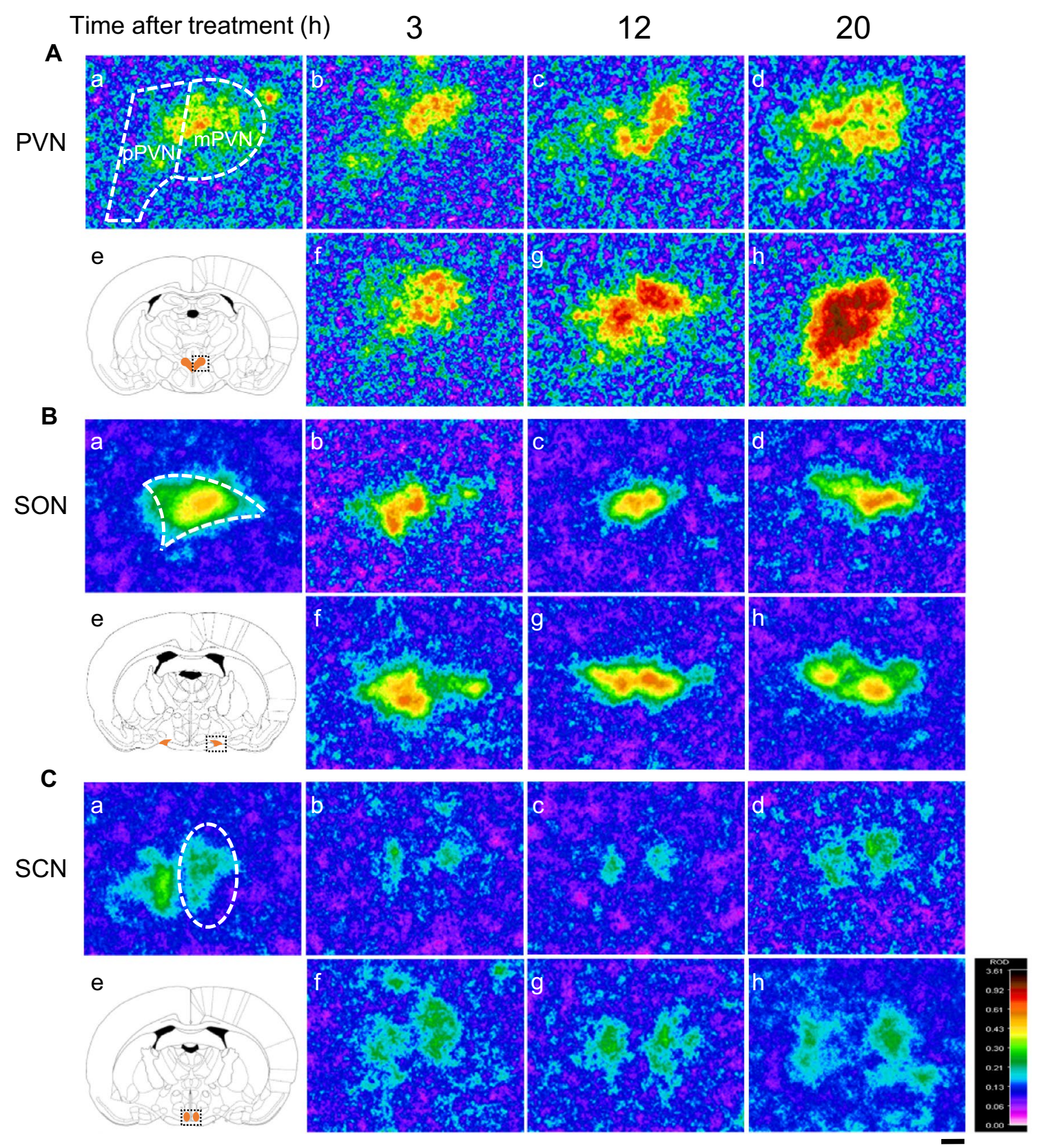

Fig. 3 Representative autoradiographs of sections hybridized with a ${ }^{35} \mathrm{~S}$-labeled oligodeoxynucleotide probe complementary to eGFP in the PVN (A), SON (B), and SCN (C) at 3, 12, and $20 \mathrm{~h}$ after bilateral nephrectomy $(\mathrm{Nx})$ or sham operation (Sham). Sections were obtained from untreated $(\mathbf{A}-\mathbf{C}-\mathrm{a})$, sham-operated $(\mathbf{A}-\mathbf{C}-\mathrm{b}-\mathrm{d})$, and

rats is considered to be similar to the change of AVP in wildtype rats. Thus, AVP-eGFP transgenic rats are beneficial transgenic animal models for investigating AVP dynamics in pPVN under stressful conditions.

We demonstrated that the number of Fos-LI cells in the LC, AP, NTS, and RVLM dramatically increased after bilateral nephrectomy. Fos, also known as c-fos, has been widely used as a marker for investigating neuronal activity bilateral-nephrectomized rats (A-C-f-h) at 3, 12, and $20 \mathrm{~h}$ after treatment. Schematic anatomic representation of the nuclei (orange shading) adapted from the rat brain atlas (A-C-e). Scale bar $1 \mathrm{~mm}$. Signal intensity ranges from high (red) to low (blue)

in the central nervous system under various physiological conditions [25-27]. We estimated that the sympathetic nervous system was activated after bilateral nephrectomy, because these nuclei are responsible for modulating sympathetic nervous system activity [7, 13, 20]. Previous studies have reported that bilateral nephrectomy increased norepinephrine levels in the hypothalamus and medulla oblongata $[28,29]$. Brainstem noradrenergic neurons 


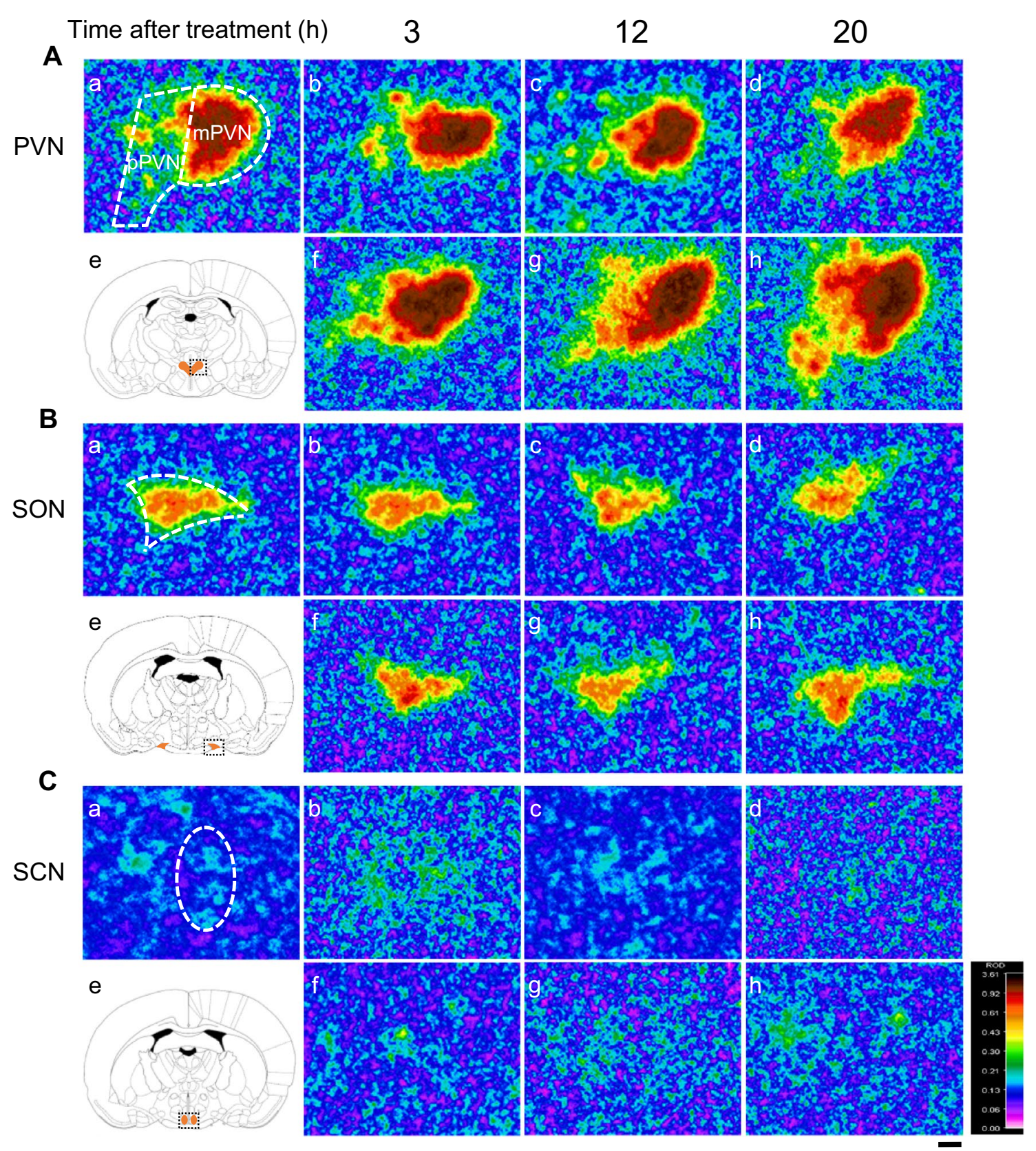

Fig. 4 Representative autoradiographs of sections hybridized with a ${ }^{35} \mathrm{~S}$-labeled oligodeoxynucleotide probe complementary to AVP in the PVN (A), SON (B), and SCN (C) at 3, 12, and $20 \mathrm{~h}$ after bilateral nephrectomy $(\mathrm{Nx})$ or sham operation (Sham). Sections were obtained from untreated $(\mathbf{A}-\mathbf{C}-\mathrm{a})$, sham-operated $(\mathbf{A}-\mathbf{C}-\mathrm{b}-\mathrm{d})$, and

mainly originating from the NTS are closely connected with HPA axis [30, 31]. Previous studies have reported that noradrenergic neural inputs differentially regulate hypothalamic AVP and CRH synthesis [32,33]. Therefore, we estimated that sympathetic nervous system activation was one of the principal factors that increased the synthesis of AVP and CRH after bilateral nephrectomy. bilateral-nephrectomized rats (A-C-f-h) at 3, 12, and $20 \mathrm{~h}$ after treatment. Schematic anatomic representation of the nuclei (orange shading) adapted from the rat brain atlas (A-C-e). Scale bar $1 \mathrm{~mm}$. Signal intensity ranges from high (red) to low (blue)

We hypothesized that there were several mechanisms underlying sympathetic nervous system activation after bilateral nephrectomy. First, previous studies have reported that autonomic nervous dysfunction occurs in patients with acute or chronic kidney disease, but the pathogenesis remains unknown [34-36]. Recent investigations have reported that uremic toxin (uric acid, indoxyl sulfate, and 


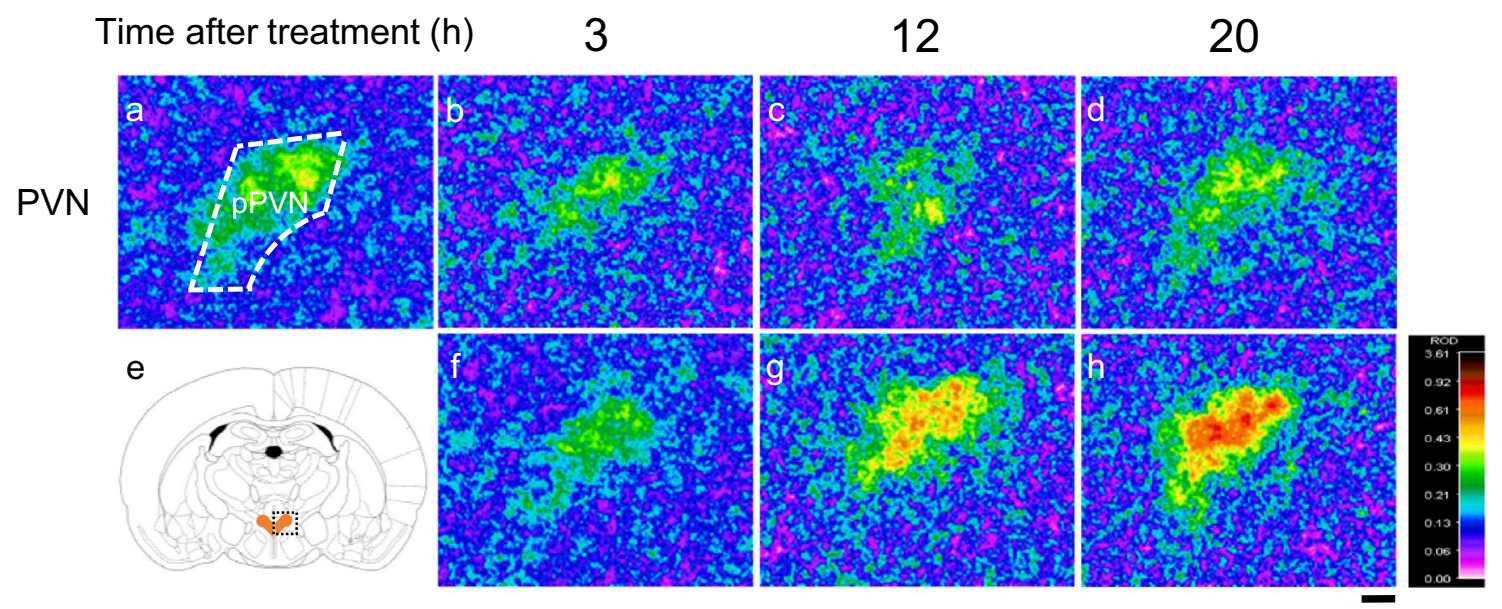

Fig. 5 Representative autoradiographs of sections hybridized with a ${ }^{35} \mathrm{~S}$-labeled oligodeoxynucleotide probe complementary to $\mathrm{CRH}$ in the PVN at 3,12, and $20 \mathrm{~h}$ after bilateral nephrectomy (Nx) or sham operation (Sham). Sections were obtained from untreated (A-a), sham-operated (A-b-d), and bilateral-nephrectomized rats (A-f-h) at 3,12 , and $20 \mathrm{~h}$ after treatment. Schematic anatomic representation of the nuclei (orange shading) adapted from the rat brain atlas (e). Scale bar $1 \mathrm{~mm}$. Signal intensity ranges from high (red) to low (blue)
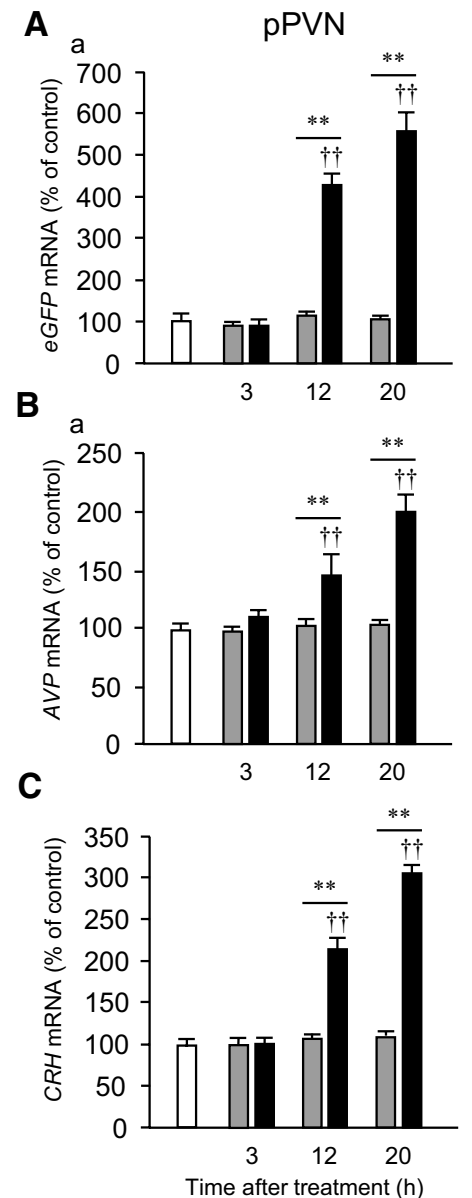

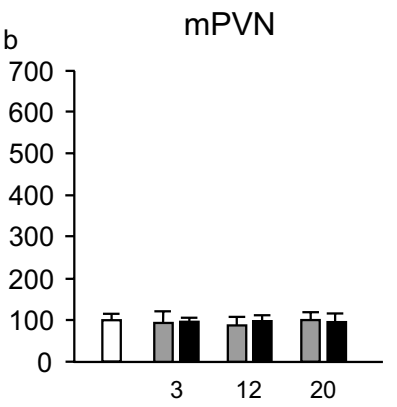

b

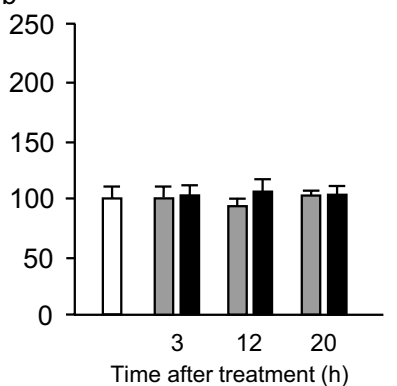

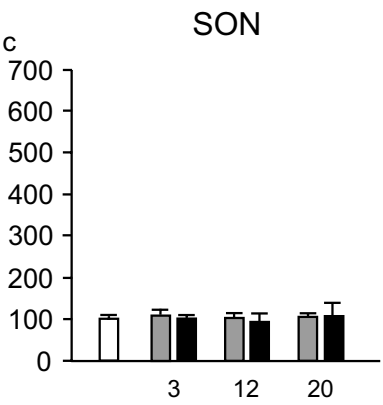

C
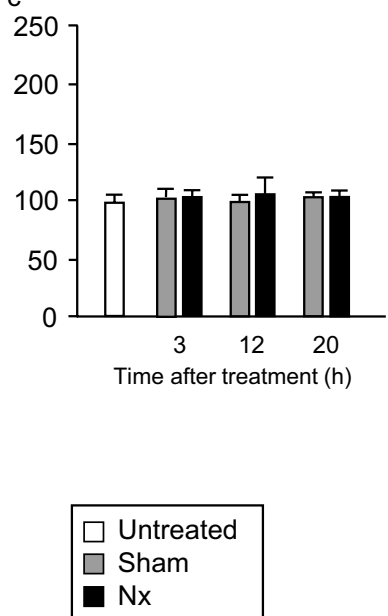

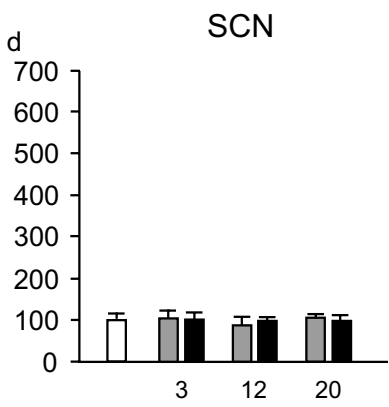

d

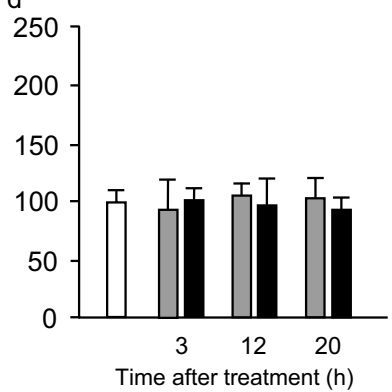

Fig. 6 Effects of bilateral nephrectomy $(\mathrm{Nx})$ or sham operation (Sham) on the gene expression of the eGFP (A), AVP (B), and $C R H$ (C) in the pPVN (A-C-a), mPVN (A, B-b), SON (A, B-c), and SCN $(\mathbf{A}, \mathbf{B}-\mathrm{d})$ in the AVP-eGFP transgenic rats. Data are presented

as the mean \pm SEM (each group at each time point, $n=6$ ). ${ }^{\dagger} P<0.05$, ${ }^{\dagger \dagger} P<0.01$ compared with the untreated group. $* P<0.05$, $* * P<0.01$ compared with the sham-operated group 


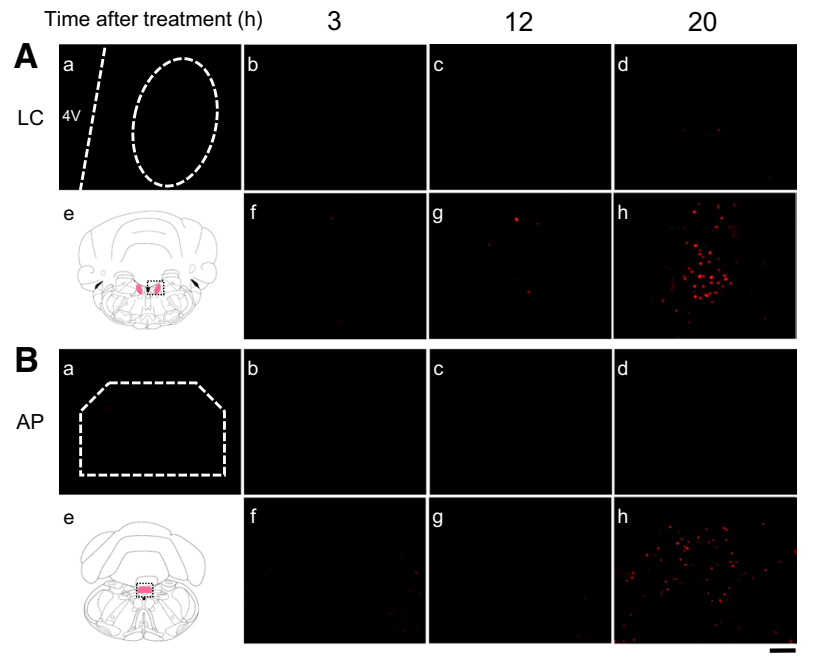

Fig. 7 Effects of bilateral nephrectomy (Nx) or sham operation (Sham) on fluorescent immunohistochemistry (FIHC) for Fos in the LC (A), AP (B), NTS (C), and RVLM (D) in the AVP-eGFP transgenic rats. Sections were obtained from untreated (A-D-a), sham-

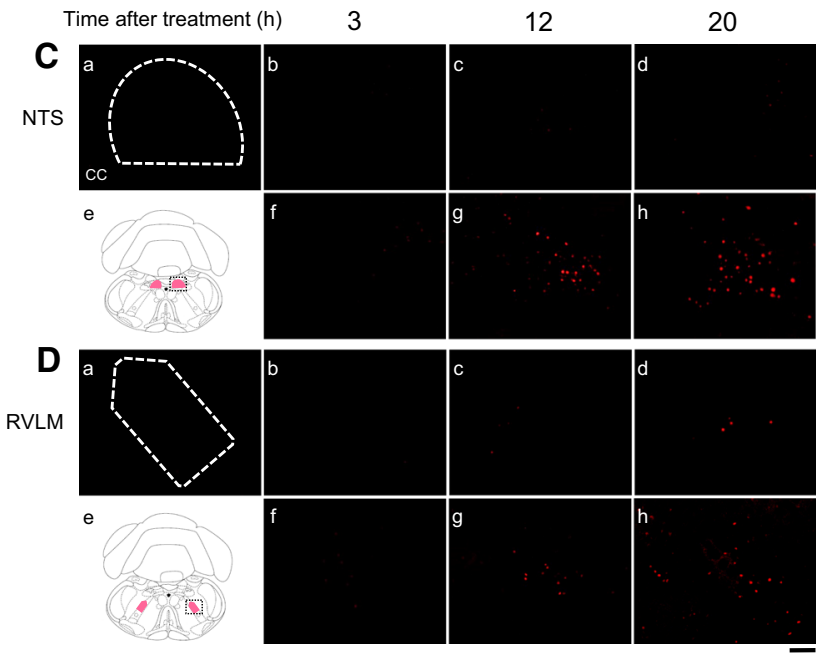

operated (A-D-b-d), and bilateral-nephrectomized rats (A-D-f-h) at 3,12 , and $20 \mathrm{~h}$ after treatment (each group at each time point, $n=6$ ). Schematic anatomic representation of the nuclei (pink shading) adapted from the rat brain atlas (A-D-e). Scale bar $100 \mu \mathrm{m}$

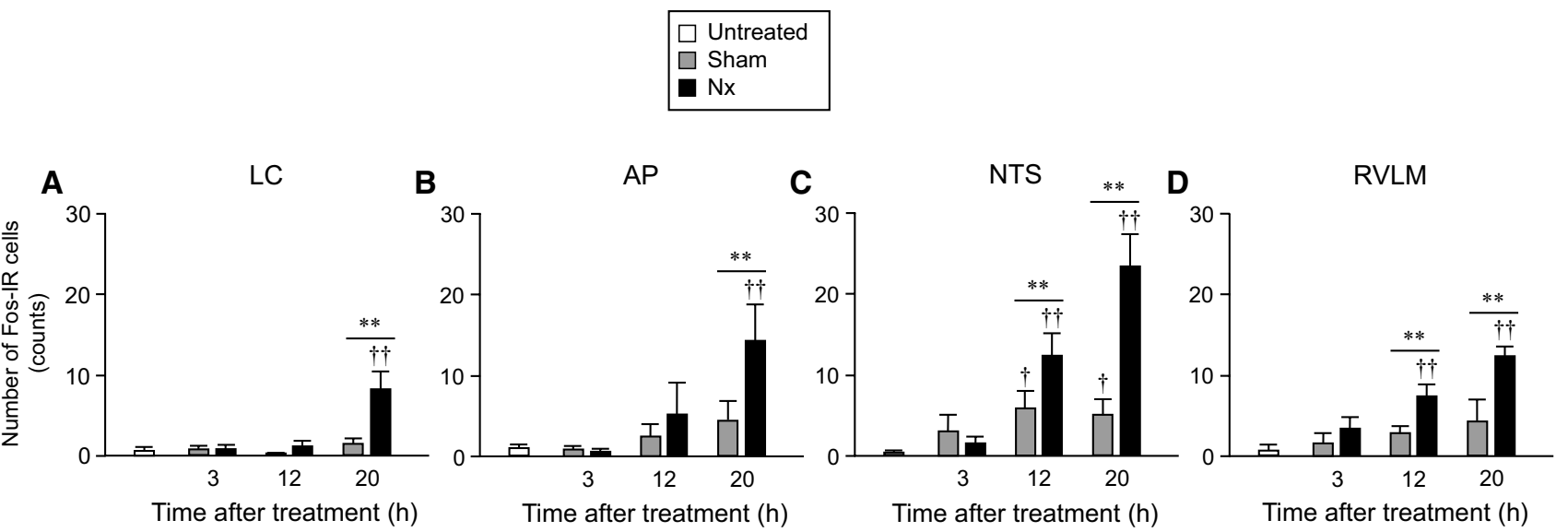

Fig. 8 Number of Fos-LI cells in the LC (A), AP (B), NTS, and RVLM (D) at 3, 12, and $20 \mathrm{~h}$ after bilateral nephrectomy (Nx) or sham operation (Sham). Data are presented as the mean \pm SEM (each

methylguanidine) increases neuronal activity in the RVLM [37]. Thus, there is a possibility that the sympathetic nervous system is activated by uremic toxin. Second, depletion of Angiotensin II (Ang II) induced by bilateral nephrectomy might play key roles in sympathetic neural activation. It has been reported that plasma renin activity and Ang II are significantly decreased after bilateral nephrectomy [38]. Fernandez et al. have reported that Ang II could modulate synthesis, uptake, and metabolism of noradrenaline in the central nervous system $[28,29,39]$. Thus, depletion of Ang II in bilateral nephrectomized rats could activate the sympathetic nervous system. Removal of the renal nerve in bilaterally nephrectomized rats may cause activation of the group at each time point, $n=6) .{ }^{\dagger} P<0.05,{ }^{\dagger} P<0.01$ compared with the untreated group. $* P<0.05, * * P<0.01$ compared with the shamoperated group

sympathetic nervous system and/or AVP neurons, because afferent renal nerve stimulation reportedly excites AVPproducing cells in the hypothalamus [40, 41]. Due to the changes in several parameters after bilateral nephrectomy, such as fluid volume and plasma electrolyte levels, there is a possibility that these factors may directly influence hypothalamic AVP dynamics. Additional experiments are needed to determine the detailed mechanisms underlying activation of the sympathetic nervous system and AVP synthesis after bilateral nephrectomy.

We should note that bilateral nephrectomy as animal models of kidney dysfunctional have a number of problems. For example, we could not evaluate the hypothalamic 
AVP dynamics for a long time after bilateral nephrectomy. The survival time of the bilateral nephrectomized rat is up to 2 days after operation, because bilateral nephrectomy-induced hyperkalemia can cause lethal arrhythmia. Therefore, animals with bilateral nephrectomy are not appropriate models for examining the effects of chronic kidney dysfunction on hypothalamic AVP synthesis and neuronal circuits. A subject of future investigations will be to determine whether a better physiological model rat of renal function degeneracy, such as 5/6 nephrectomy and renal ischemia reperfusion injury, will show upregulation of hypothalamic AVP synthesis and activation of the sympathetic nervous system.

\section{Conclusions}

The HPA axis has a key role in maintaining homeostasis under several stress conditions. The biological response to stress is mediated by the regulation of complex systems, such as the nervous, endocrine, and immune systems. AVP and CRH are principal factors in the endocrine system for biological responses to several stressors. Previous studies have reported that the upregulation of AVP and CRH synthesis mainly depends on the complex neural pathway to the PVN from the brainstem NE neurons. In conclusion, we were able to successfully use transgenic rats to reveal the upregulation of hypothalamic parvocellular AVP after bilateral nephrectomy. In addition, Fos-LI expression in brainstem neurons revealed that bilateral nephrectomy might activate the sympathetic nervous system. These findings provide novel insights into the hypothalamic response in stress conditions induced by acute kidney dysfunction. Additional studies are needed to identify the neural and humoral factors that activate the central nervous system after bilateral nephrectomy.

Acknowledgements We express our appreciation to Ms. Yuki Nonaka for her technical assistance.

Author contributions Conception or design of the work; HU, RS, TM, MT, YO, YU. Acquisition, analysis, or interpretation of data for the work; HU, KS, YA, KT, HN, KN, SS, YM, RS, MY, TM. Drafting the work or revising it critically for important intellectual content; HU, RS, KS, YA, KT, HN, KN, SS, YM, RS, MY, TM, MT, YO, YU. All authors approved the final version of the manuscript and agree to be accountable for all aspects of the work in ensuring that questions related to the accuracy or integrity of any part of the work are appropriately investigated.

Funding This paper was supported by a Grant-in-Aid for Scientific Research (B) (No. 17H04027), (C) (No. 17K08582), and Young Scientist (B) (No. 17K15575) from the Japan Society for the Promotion of Science (JSPS).

\section{Compliance with ethical standards}

Conflict of interest The authors declare that they have no conflict of interest.

Ethical approval All procedures in this study were performed in accordance with the guidelines on the use and care of the laboratory animals as set out care by the Physiological Society of Japan and under control of the Ethics Committee of Animal Care and Experimentation, University of Occupational and Environmental Health (Fukuoka, Japan).

\section{References}

1. Ueno H, Yoshimura M, Tanaka K et al (2018) Up-regulation of hypothalamic arginine vasopressin by peripherally administered furosemide in transgenic rats expressing arginine vasopressinenhanced green fluorescent protein. J Neuroendocrinol 30:1-16. https://doi.org/10.1111/jne. 12603

2. Suzuki H, Onaka T, Kasai M et al (2009) Response of arginine vasopressin-enhanced green fluorescent protein fusion gene in the hypothalamus of adjuvant-induced arthritic rats. J Neuroendocrinol 21:183-190. https://doi.org/10.111 1/j.1365-2826.2009.01841.x

3. Shibata M, Fujihara H, Suzuki H et al (2007) Physiological studies of stress responses in the hypothalamus of vasopressin-enhanced green fluorescent protein transgenic rat. J Neuroendocrinol 19:285-292. https://doi.org/10.1111/j.1365-2826.2007.01532.x

4. Sofroniew MV, Weindl A (1980) Identification of parvocellular vasopressin and neurophysin neurons in the suprachiasmatic nucleus of a variety of mammals including primates. J Comp Neurol 193:659-675. https://doi.org/10.1002/cne.901930305

5. Uhl GR, Reppert SM (1986) Suprachiasmatic nucleus vasopressin messenger RNA: circadian variation in normal and Brattleboro rats. Science 232:390-393

6. Bartanusz V, Jezova D, Bertini LT et al (1993) Stress-induced increase in vasopressin and corticotropin-releasing factor expression in hypophysiotrophic paraventricular neurons. Endocrinology 132:895-902. https://doi.org/10.1210/endo.132.2.8425502

7. Bisset GW, Chowdrey HS (1988) Control of release of vasopressin by neuroendocrine reflexes. Q J Exp Physiol 73:811-872. https:// doi.org/10.1113/expphysiol.1988.sp003223

8. Suzuki H, Kawasaki M, Ohnishi H et al (2009) Exaggerated response of a vasopressin-enhanced green fluorescent protein transgene to nociceptive stimulation in the rat. $\mathrm{J}$ Neurosci 29:13182-13189. https://doi.org/10.1523/JNEUR OSCI.2624-09.2009

9. Fujio T, Fujihara H, Shibata M et al (2006) Exaggerated response of arginine vasopressin-enhanced green fluorescent protein fusion gene to salt loading without disturbance of body fluid homeostasis in rats. J Neuroendocrinol 18:776-785. https://doi.org/10.111 1/j.1365-2826.2006.01476.x

10. Ueta Y, Fujihara H, Serino R et al (2005) Transgenic expression of enhanced green fluorescent protein enables direct visualization for physiological studies of vasopressin neurons and isolated nerve terminals of the rat. Endocrinology 146:406-413. https:// doi.org/10.1210/en.2004-0830

11. McKinley MJ, Mathai ML, McAllen RM et al (2004) Vasopressin secretion: osmotic and hormonal regulation by the lamina terminalis. J Neuroendocrinol 16:340-347. https://doi.org/10.111 1/j.0953-8194.2004.01184.x 
12. Dos Santos Moreira MC, Naves LM, Marques SM et al (2017) Neuronal circuits involved in osmotic challenges. Physiol Res 8408:411-423

13. Cruz JC, Bonagamba LGH, Machado BH et al (2008) Intermittent activation of peripheral chemoreceptors in awake rats induces Fos expression in rostral ventrolateral medulla-projecting neurons in the paraventricular nucleus of the hypothalamus. Neuroscience 157:463-472. https://doi.org/10.1016/j.neuroscience.2008.08.070

14. Kawai S, Takagi Y, Kaneko S, Kurosawa T (2011) Effect of three types of mixed anesthetic agents alternate to ketamine in mice. Exp Anim 60:481-487. https://doi.org/10.1538/expanim.60.481

15. Motojima Y, Matsuura T, Yoshimura M et al (2017) Comparison of the induction of c-fos-eGFP and Fos protein in the rat spinal cord and hypothalamus resulting from subcutaneous capsaicin or formalin injection. Neuroscience 356:64-77. https://doi. org/10.1016/j.neuroscience.2017.05.015

16. Paxinos G, Watson C (2005) The rat brain in stereotaxic coordinates. Elsevier Academic Press, Amsterdam

17. Arase K, Hashimoto H, Sonoda S et al (2018) Possible involvement of central oxytocin in cisplatin-induced anorexia in rats. J Physiol Sci 68:471-482. https://doi.org/10.1007/s1257 6-017-0550-z

18. Morita H, Yamaguchi A, Shiba D et al (2017) Impact of a simulated gravity load for atmospheric reentry, $10 \mathrm{~g}$ for $2 \mathrm{~min}$, on conscious mice. J Physiol Sci 67:531-537. https://doi.org/10.1007/ s12576-017-0526-Z

19. So M, Hashimoto H, Saito R et al (2018) Inhibition of ghrelininduced feeding in rats by pretreatment with a novel dual orexin receptor antagonist. J Physiol Sci 68:129-136. https://doi. org/10.1007/s12576-016-0517-5

20. Ueta Y, Yamashita H, Kawata M, Koizumi K (1995) Water deprivation induces regional expression of $\mathrm{c}$-fos protein in the brain of inbred polydipsic mice. Brain Res 677:221-228

21. Yoshimura M, Hagimoto M, Matsuura T et al (2014) Effects of food deprivation on the hypothalamic feeding-regulating peptides gene expressions in serotonin depleted rats. J Physiol Sci 64:97104. https://doi.org/10.1007/s12576-013-0296-1

22. Kinoshita H, Jessop DS, Finn DP, Harbuz MS (2000) Cyanamideinduced activation of the hypothalamo-pituitary-adrenal axis. $\mathbf{J}$ Neuroendocrinol 12:255-262

23. Cell Zeng Q, Carter DA, Murphy D (1994) Cell specific expression of a vasopressin transgene in rats. J Neuroendocrinol 6:469-477

24. Itagaki E, Ozawa S, Yamaguchi $S$ et al (2001) Increases in plasma ACTH and cortisol after hypertonic saline infusion in patients with central diabetes insipidus. J Clin Endocrinol Metab 86:5749_ 5754. https://doi.org/10.1210/jcem.86.12.8073

25. Morgan JI, Cohen DR, Hempstead JL, Curran T (1987) Mapping patterns of c-fos expression in the central nervous system after seizure. Science 237:192-197

26. Cunningham JT, Grindstaff RJ, Grindstaff RR, Sullivan MJ (2002) Fos immunoreactivity in the diagonal band and the perinuclear zone of the supraoptic nucleus after hypertension and hypervolaemia in unanaesthetized rats. J Neuroendocrinol 14:219-227

27. Curran T, Morgan JI (1995) Fos: an immediate-early transcription factor in neurons. J Neurobiol 26:403-412. https://doi. org/10.1002/neu.480260312
28. Fernandez BE, Dominguez AE (1990) Effects of angiotensin II and bilateral nephrectomy on norepinephrine catabolism in central nervous system. Arch Int Physiol Biochim 98:307-313

29. Domínguez AE, Fernández BE, Vidal NA, Martínez Seeber A (1982) Angiotensin II-norepinephrine relationship in the central nervous system. Arch Int Physiol Biochim 90:269-275

30. Plotsky PM (1987) Facilitation of immunoreactive corticotropinreleasing factor secretion into the hypophysial-portal circulation after activation of catecholaminergic pathways or central norepinephrine injection. Endocrinology 121:924-930. https://doi. org/10.1210/endo-121-3-924

31. Kiss A, Aguilera G (1993) Regulation of the hypothalamic pituitary adrenal axis during chronic stress: responses to repeated intraperitoneal hypertonic saline injection. Brain Res 630:262-270

32. Itoi K, Helmreich DL, Lopez-Figueroa MO, Watson SJ (1999) Differential regulation of corticotropin-releasing hormone and vasopressin gene transcription in the hypothalamus by norepinephrine. J Neurosci 19:5464-5472

33. Helmreich DL, Itoi K, Lopez-Figueroa MO et al (2001) Norepinephrine-induced $\mathrm{CRH}$ and AVP gene transcription within the hypothalamus: differential regulation by corticosterone. Mol Brain Res 88:62-73. https://doi.org/10.1016/S0169-328X(01)00018-3

34. Campese VM, Romoff MS, Levitan D et al (1981) Mechanisms of autonomic nervous system dysfunction in uremia. Kidney Int 20:246-253

35. Vita G, Bellinghieri G, Trusso A et al (1999) Uremic autonomic neuropathy studied by spectral analysis of heart rate. Kidney Int 56:232-237. https://doi.org/10.1046/j.1523-1755.1999.00511.x

36. Hering D, Winklewski PJ (2017) R1 autonomic nervous system in acute kidney injury. Clin Exp Pharmacol Physiol 44:162-171. https://doi.org/10.1111/1440-1681.12694

37. Oshima N, Onimaru H, Matsubara $\mathrm{H}$ et al (2015) Uric acid, indoxyl sulfate, and methylguanidine activate bulbospinal neurons in the RVLM via their specific transporters and by producing oxidative stress. Neuroscience 304:133-145. https://doi. org/10.1016/j.neuroscience.2015.07.055

38. Ferrario CM, Varagic J, Habibi J et al (2009) Differential regulation of angiotensin-(1-12) in plasma and cardiac tissue in response to bilateral nephrectomy. Am J Physiol Heart Circ Physiol 296:H1184-H1192. https://doi.org/10.1152/ajpheart.01114.2008

39. Domínguez AE, Fernández BE, Vidal NA (1983) The renin-angiotensin system and noradrenaline release in the hypothalamus and medulla oblongata. Rev Esp Fisiol 39:249-252

40. Day TA, Ciriello J (1987) Effects of renal receptor activation on neurosecretory vasopressin cells. Am J Physiol 253:R234-R241

41. Simon JK, Kasting NW, Ciriello J (1989) Afferent renal nerve effects on plasma vasopressin and oxytocin in conscious rats. Am J Physiol 256:R1240-R1244

Publisher's Note Springer Nature remains neutral with regard to jurisdictional claims in published maps and institutional affiliations. 